\title{
Sleep and aging:
}

Examining the effect of psychological symptoms and risk of sleep-disordered breathing

\author{
Alix Mellor ${ }^{1,2 *}$, Flavie Waters ${ }^{1,2,4}$, Michelle Olaithe ${ }^{3}$, Helen McGowan ${ }^{4}$, Romola S. Bucks ${ }^{3}$ \\ ${ }^{1}$ School of Psychiatry and Clinical Neurosciences, University of Western Australia, Perth \\ ${ }^{2}$ Clinical Research Centre, Graylands Hospital, North Metropolitan Mental Health, Perth \\ ${ }^{3}$ School of Psychology, University of Western Australia, Perth \\ ${ }^{4}$ Older Adult Clinical Research Unit, North Metropolitan Mental Health, Perth
}

\begin{abstract}
*Corresponding author: Clinical Research Centre, Graylands Hospital, Private Bag, No. 1, Claremont WA, 6910, Ph +61 89347 6418, Fax +61 89384 5128, Email address: mellora@meddent.uwa.edu.au
\end{abstract}

Journal: Behavioral Sleep Medicine

Total word count: 3913

Running title: Sleep and aging 


\begin{abstract}
Controversy exists as to whether self-reported sleep quality declines with age, despite changes in sleep being accepted as part of normal aging. This study sought to investigate age-related differences in self-reported sleep quality, after controlling for conditions that are common with age, such as psychological symptoms and increased risk of sleep-disordered breathing (SDB). The Pittsburgh Sleep Quality Index (PSQI) was administered to a sample of 582 community adults (18-89 years), and the association between age and three factors of the PSQI (Sleep Efficiency, Perceived Sleep Quality, Daily Disturbance), and global scores, was examined controlling for depression, anxiety, stress, gender and SDB-risk. Results indicate that: (i) Before controlling for covariates, there was no significant relationship between age and all indices of self-reported sleep quality, with the exception of Sleep Efficiency. However, once depression, gender and SDB-risk were controlled for, a significant, yet small relationship was revealed between older age and poorer global sleep quality; (ii) there was no association between age and Perceived Sleep Quality, or Daily Disturbances, before or after controlling for relevant covariates; and (iii) depression, gender and SDB-risk were significant predictors of poorer sleep quality across the indices, but in general, did not have a marked impact on the relationship between age and sleep quality. In conclusion, results suggest that sleep problems are common across the lifespan, and that there were modest age-related differences in self-reported sleep quality, which were not due to depressed mood, gender, or risk of SDB.
\end{abstract}




\section{Introduction}

Changes in sleep are part of the normal aging process, and include alterations to sleep architecture and sleep-wake patterns, which have been reported using objective measures of sleep such as polysomnography (PSG) and actigraphy. The timing of sleep is typically shifted towards early bed and rise times, and there are increased nighttime awakenings. In addition, sleep efficiency and the percentage of slow-wave sleep are reduced (Knowles \& MacLean, 1990; McCrae, 2009; Ohayon, Carskadon, Guilleminault, \& Vitiello, 2004). However, controversy exists as to whether self-reported sleep quality, commonly assessed using questionnaires such as the Pittsburgh Sleep Quality Index (PSQI; Buysse, Reynolds, Monk, Berman, \& Kupfer, 1989), also declines with age.

Several large epidemiological studies that assessed sleep across the lifespan (18-65+ years of age) have reported that age is linked to an increase in subjective sleep complaints. These include difficulties with sleep maintenance and reduced sleep duration (e.g., Stein, Belik, Jacobi, \& Sareen, 2008; Zeitlhofer et al., 2000). By contrast, other studies of older adults (>60 years of age), with equally large samples, have found that older adults do not necessarily rate their sleep quality as poor and are largely satisfied with their sleep once health conditions linked to aging have been controlled for (Foley et al., 1995; Vitiello, Moe, \& Prinz, 2002). Indeed, several studies have reported that subjective sleep quality in older adults was not significantly different from that of middle-aged adults (e.g., Middelkoop, Smilde-van den Doel, Neven, Kamphuisen, \& Springer, 1996), and a recent study of adults aged 18-80+ years even found that older adults were less likely to report poorer sleep quality and tiredness than younger adults (Grandner et al., 2012). 
One reason for such mixed findings could be that older people adjust their expectations about their sleep as they age (Buysse et al., 1991; Zilli, Ficca, \& Salzarulo, 2009). Methodological issues might also explain these seemingly contradictory findings. For example, differences exist between studies in the aspect of sleep quality being investigated, and in the instruments employed to assess it. Sleep quality is a multifaceted concept that includes individual components such as satisfaction with sleep, sleep efficiency, and impact on daytime functioning (Magee, Caputi, Iverson, \& Huang, 2008). Many epidemiological studies have used global scores from the PSQI to demonstrate age-related decline in sleep quality (e.g., Stein et al., 2008; Wong \& Fielding, 2011; Zeitlhofer et al., 2000), although recent factor analytic studies suggest that the PSQI has a multidimensional structure that is better characterized by a two- or threefactor model (Cole et al., 2006; Magee et al., 2008). Specific associations might, therefore, exist between age and PSQI sleep factors, but not with global scores. This suggests that the characterization of different dimensions of sleep must remain an important focus in studies of sleep quality across the lifespan.

Psychological factors, such as depression, have also been cited as important factors that can affect the relationship between sleep quality and age. A large study by Roberts, Shema, and Kaplan (1999), for instance, showed that the trend for increased sleep disturbance across the lifespan (specifically assessed with symptoms of insomnia and hypersomnia) disappeared when levels of negative mood were controlled for. This finding is consistent with cross-sectional and longitudinal studies documenting the reciprocal relationship between sleep problems and psychological problems such as depression (Krishnan \& Hawranik, 2008; Sbarra \& Allen, 2009; Taylor, Lichstein, Durrence, Reidel, \& Bush 2005; Walker \& Van der Helm, 2009). 
One possibility exists, therefore, that sleep problems in older adults may be directly linked to psychological symptoms such as depression, anxiety, or stress, rather than aging per se. However, a recent study by Grandner et al. (2012) contradicts the suggestion that controlling for depression might impact on the way that age impacts on sleep quality. The authors adjusted their results for depression and found that age-related changes in self-reported sleep quality remained, despite the trend being in the opposite direction to the results reported by Roberts et al. (1999). That is, sleep disturbances decreased, rather than increased, across the lifespan despite controlling for depression. These findings suggest that depression might not have a direct impact on the relationship between age and self-reported sleep quality.

Given these discrepant results, an independent examination of this issue is warranted. In addition, with the exception of Grandner et al., few studies have examined the effect of depression, or indeed other psychological symptoms such as anxiety or stress, on sleep quality and age (Doi, Minowa, \& Tango, 2003; Hall et al., 2000). In the current study, we sought to examine whether depression, anxiety and stress contributed to age-related differences in sleep quality as measured with the PSQI.

It is important to note that the relationship between sleep, depression and age is complex. Some studies have reported that the susceptibility to depression tends to decrease, rather than increase, with age (Jorm, 2000; Henderson et al., 1998). This challenges our understanding that poor sleep in older adults should be linked to greater levels of depression and suggests that there could be differential associations between depression and sleep at different ages (Knowles \& MacLean, 1990). However, as O’Connor (2006) suggests, findings of an apparent reduction in the prevalence of depression in older adults could be due to sampling biases, such that depression is actually highly prevalent in aged individuals (Fiske, Loebach, \& Wetherell, 2009). Investigation 
into self-reported sleep quality across the lifespan, taking into account the possible effects of psychological symptoms such as depression, anxiety and stress, is therefore urgently needed to advance knowledge in this area.

Another potential confound that is often overlooked is the risk of sleep-disordered breathing (SDB). This under-diagnosed disorder includes a range of sleep-related respiratory abnormalities such as repeated episodes of high-resistance breathing, reduction in airflow and temporary cessation of breathing (Peppard, Szklo-Coxe, Hla, \& Young, 2006). These symptoms increase with age and can dramatically affect sleep quality, with reports of restless and un-refreshed sleep (Amra, Farajzadegan, Golshan, Fietze, \& Penzel, 2011; Flemons \& Tsai, 1997). In addition, the association between SDB and depression, although complex, has been well established (Harris, Glozier, Ratnavadivel, \& Grunstein, 2009). Therefore, given its links to sleep quality, age, and depression, risk of SDB should be accounted for when investigating the relationship between age and sleep quality.

In summary, the current study aimed to investigate age-related differences in sleep quality as assessed using global PSQI scores and PSQI factors, controlling for depression, anxiety and stress, and risk of SDB. In addition, gender was controlled for, since women tend to report more sleep problems than men (Voderholzer, Al-Shajlawi, Weske, Feige, \& Riemann, 2003).

\section{Method}

\section{Participants}

Recruitment was conducted via online advertising through universities, international survey and social networking sites, through advertisement in Australian community centres, and advertisement emails sent to individuals from volunteer groups (Western Australian Participant 
Pool, Director RS Bucks; and Older Adult Clinical Research Unit, H McGowan, F Waters).

Once interest was expressed, participants were either mailed a questionnaire or sent a link to the questionnaire online. Completion of the questionnaire was taken to indicate consent. All mailed questionnaires were completed and returned. In total, 615 participants completed the questionnaires. Only questionnaires completed by individuals over the age of 18 and from English-speaking and industrialized countries were used in the study. After excluding participants from non-English speaking countries and those under 18 years of age, data were analysed for 582 adults aged 18-89 years. The majority (559; 96.0\%) of participants completed the online version of the questionnaire, whilst 23 (4.0\%) completed the hardcopy version. Participants were recruited from three countries: Australia $(n=471)$; the United Kingdom ( $n=$ 31); and the United States of America $(\mathrm{n}=80)$. There were no significant differences on BMI ( $p$ $=.205)$ or gender $(p=.320)$ between participants from different countries of origin. However, there were significant differences on global sleep quality scores $(p=.004)$, hence country of origin was initially included as a covariate in the analyses. Approval was granted by both the North Metropolitan Mental Health Services and the University of Western Australia Human Research Ethics Committees.

\section{Materials}

Participants completed a demographics questionnaire, which asked about age, gender, medical history, education and occupation. They also completed the following scales:

The Pittsburgh Sleep Quality Index (PSQI; Buysse et al., 1989), which assesses sleep quality in the previous month across seven domains (sleep duration, sleep disturbance, sleep latency, daytime dysfunction, sleep efficiency, subjective sleep quality, and use of sleep medication). Component scores are calculated on the basis of raw scores (scores range from 0-3, with higher 
scores indicating poorer sleep). Scores $>5$ indicate significant sleep problems (Buysse et al., 1989). The PSQI has good estimates of reliability in a range of populations, including older adults, with alpha values quoted between .77-.83 (Beck, Schwartz, Towsley, Dudley, \& Barsevick, 2004; Carpenter \& Andrykowski, 1998; Gentili, Weiner, Kubchibhatla, \& Edinger, 1995; Spira et al., 2012). In this study, Cronbach’s alpha was .71 for the global score and .61 for the 9-item sleep disturbance subscale. As reported by Buysse et al. (1989), alpha values were not calculated for the other subscales due to the small number of items, rather correlations between each of the seven subscales and the global score were computed. These were all significant (all $p$ $<.001$ ), ranging from .37-.68, which compared well to the original article that reported coefficients between .35-.76. This suggests that although the PSQI has good reliability overall, the global score may not be a robust measure of sleep quality, as suggested by Cole et al. (2006). Accordingly, Cole and colleagues derived a three-factor model of the PSQI: Sleep Efficiency (comprising PSQI components: sleep efficiency and sleep duration), Perceived Sleep Quality (comprising PSQI components: use of sleep medication, sleep latency, and subjective sleep quality), and Daily Disturbances (comprising PSQI components: daytime dysfunction and sleep disturbance). This model has been used in a range of community studies with young and older adults, and in people with chronic fatigue syndrome (Aloba, Adewuya, Ola, \& Mapayi, 2007; Cole et al., 2006; Mariman et al., 2012).

The Depression Anxiety Stress Scale (DASS-21; Lovibond \& Lovibond, 1995), which measures the frequency of these core negative symptoms over the past week. There are seven questions per subscale and scores for each subscale range from $0-21$, with higher scores indicating more symptoms. Internal consistency was high, $\alpha=.94$ overall; Depression: $\alpha=.91$; Anxiety: $\alpha=.82$; 
and Stress: $\alpha=.87$. These values are consistent with previous studies using community samples, which also have excellent reliability (Crawford et al., 2009; Crawford \& Henry, 2005).

The Berlin Questionnaire (BQ; Netzer, Stoohs, Netzer, Clark, \& Strohl, 1999) is a self-report measure, which assesses risk of SDB. It includes questions about snoring and breathing during sleep (Category 1), feelings of fatigue and tiredness after sleep (Category 2), and BMI (which is calculated from height and weight), and whether people experience high blood pressure (Category 3). Risk of SDB was based on the following criteria: For Category 1, persistent symptoms (3-4 times a week) for two or more questions about snoring; for Category 2, persistent feelings of sleepiness during the day, and/or drowsiness during driving (3-4 times a week); and for Category 3, a history of hypertension ('yes', ‘no’, ‘don’t know’), or a BMI>30. Only one response to either of these questions is needed to score this category as positive, and 'don't know' responses are scored as negative. Whilst some participants did not know their height and weight $(n=50)$, these individuals all scored at risk for Category 3 on the basis of the other question. Two or more positive responses across the three categories indicate a high risk of SDB. The BQ has high internal validity (Cronbach’s alpha estimates range from .86-.92; Netzer et al., $1999 ;$ and $\alpha=.59$ in this study).

\section{Statistical analysis}

Data were analyzed using SPSS Version 20 (IBM, Inc). There were few missing PSQI data (3.3\%; number of missing values / number of variables * number of participants), which came from the hardcopy questionnaires. Formatting of the online questionnaire did not allow participants to miss questions. Because the data was missing at random, Estimation Maximization was used to replace missing values on PSQI subscales (Field, 2009). Relationships 
between study variables were investigated using Spearman's correlation coefficients. The analyses included the PSQI global score and PSQI factors (Sleep Efficiency, Perceived Sleep Quality, and Daily Disturbances; Cole et al., 2006). Factor scores were calculated for each participant by multiplying the relevant component scores comprising each factor by the factor loadings presented by Cole et al. (2006), and summing them.

Multiple Regression Analyses were used to examine the effect of age on PSQI variables (global scores and the three factors). Given that gender, depression, anxiety and stress correlated significantly with age and PSQI factors, initially these variables were entered as covariates in the analyses. Similarly, given a significant age-difference between those at risk, and those not at risk of SDB, as indicated by a $t$-test, SDB-risk was entered as a covariate in the analyses. Anxiety and stress (measured with the DASS-21) were not significant predictors of the PSQI factors and were not included in the final regression models. Similarly, country of origin (dummy coded as AUS vs UK/US and US vs AUS/UK) was not a significant predictor of any sleep variables and was therefore not included in the final regression models.

\section{Results}

Table 1 reports descriptive statistics for the demographics and clinical questionnaire for the sample. Over $20 \%$ of the sample was characterized as 'at risk' of SDB as assessed by the Berlin Questionnaire (BQ; see Table 1).

----- Insert Table 1 here -----

----- Insert Table 2 here ----- 
Table 2 presents the descriptive statistics for the PSQI global and factor scores for the whole sample, and by decade of life ${ }^{1}$. Half the respondents $(N=306 ; 52.3 \%)$ scored $>5$ on the global PSQI score, indicating significant sleep problems (Buysse et al., 1989).

----- Insert Table 3 here -----

Table 3 reports bivariate Spearman's correlations between PSQI global and factor scores, and age, depression, anxiety and stress (DASS-21). There was no significant correlation between age and global PSQI scores. Similarly, there was no relationship between age and Perceived Sleep Quality, or Daily Disturbances. However, there was a significant positive, yet weak, correlation between age and Sleep Efficiency (higher scores indicate more disturbance; $r h o=.16, p<.01$ ). In addition, increasing age was associated with less depression, lower anxiety and less stress, although those at risk of SDB were likely to be older, $t(578)=-5.43, p<.001$.

----- Insert Table 4 here -----

We conducted four separate multiple regression analyses and as expected, gender, depression and risk of SDB were significant covariates across the sleep quality indices. Depression was linked to poorer sleep quality on global scores, Sleep Efficiency, Perceived Sleep Quality, and Daily Disturbances ( $\beta=.18$ to $.37, p<.01)$. Anxiety and stress were not related to sleep quality. Risk of SDB was linked to poorer sleep quality on global scores, Sleep Efficiency, and Daily Disturbances ( $\beta=.13$ to $.18, p<.01$ ). Similarly, gender was a significant predictor of global sleep quality, Perceived Sleep Quality, and Daily Disturbances, with females reporting poorer sleep quality than males ( $\beta=.11$ to $.13, p<.01)$. Therefore, these results confirm the need to control for gender, depression and risk of SDB in the regression analyses (Table 4).

\footnotetext{
${ }^{1}$ One reviewer expressed concern about the disproportionate number of participants in the 18-29 year age bracket, which could have skewed results. Accordingly, we randomly selected 80 participants from the 18-29 year age bracket and reran all analyses; the results did not change.
} 
Despite the significant impact of depression, SDB-risk and gender on sleep quality, there was no substantive effect of these covariates on the relationship between age and the indices of sleep quality (see Table 4). Relationships between age and the three factors of the PSQI did not change once gender, depression and SDB-risk were controlled for. That is, there was no relationship between age and Perceived Sleep Quality, or Daily Disturbances before or after controlling for relevant covariates. Further, there was a significant relationship between age and Sleep Efficiency before and after controlling for risk of SDB and depression, however the magnitude of this effect was very small and must not be over-interpreted ( $\mathrm{R}$ values between $.01-.089$ were considered small effects, those between .09-.249 as medium, and those greater than .25 as large effects; Cohen, 1988). For PSQI global scores, a significant age-effect was revealed after controlling for relevant covariates, such that older age was associated with poorer overall sleep quality (gender, depression and risk of SDB; see Table 4) - however, the association between age and global sleep scores was of very modest strength and must, therefore, be interpreted with caution (Cohen, 1988).

\section{Discussion}

This study investigated the relationship between age and self-reported sleep quality, and whether factors such as depression, anxiety, stress and risk of SDB affected this relationship. Overall, findings indicate that: (i) after controlling for gender, depression and SDB-risk, there was a modest association between age and global sleep scores, which was largely driven by age differences in Sleep Efficiency, (ii) there was no relationship between age and Perceived Sleep Quality, and Daily Disturbances before and after controlling for relevant covariates; (iii) depression, gender and risk of SDB were significant predictors of some indices of sleep quality, 
but did not have a marked impact on the relationship between age and self-reported sleep quality. In summary, results suggest little age-related difference in self-reported sleep quality.

Prevalence of sleep problems in our sample

Overall, the prevalence of sleep problems in our sample was relatively high (52.3\%). The global PSQI score in the current study ( $\mathrm{M}=6.4, \mathrm{SD}=3.3)$ was slightly higher than in other community studies, although still within one standard deviation of larger studies (e.g., Wong \& Fielding, 2011, $M$ = 5.3, SD = 3.3; Stein et al., 2008, $M=$ 5, SD = 3; Zeitlhofer et al., 2000, $M=4.6$, SD = 3.7), suggesting that our sample was broadly representative of community adults.

The effect of psychological factors on the relationship between age and sleep quality

This study investigated how psychological factors, including depression, anxiety and stress, affected the relationship between age and sleep quality. Firstly, results indicate that depression, anxiety and stress decreased with age. These results are consistent with other studies of older adults recruited from the community (rather than care homes) that show that the risk of depression decreases with age (e.g., Jorm, 2000). Secondly, contrary to previous reports reporting that psychological factors such as depression may explain sleep difficulties associated with aging (e.g., Roberts et al., 1999), current results do not support this proposition. While depression was associated with reduced sleep quality, it was not linked to age-related sleep problems. This suggests that controlling for symptoms of depression had little impact on the relationship between age and sleep quality, as recently reported by Grandner et al (2012). 
The effect of SDB-risk on the relationship between age and sleep quality

In the current study, SDB-risk increased with age and depression, as previously reported (Amra et al., 2011). Increased risk of SDB was also associated with poorer sleep quality, however results indicate that it had largely no impact on the relationship between age and sleep quality. While this suggests that SDB-risk had little explanatory power in the relationship between sleep and age, the current study requires independent replication in a clinical sample of individuals with SDB, given the low alpha value for the Berlin Questionnaire (.59) in this community sample.

Age and sleep quality, and association with covariates (gender, psychological factors and SDBrisk)

Initial analysis of PSQI global scores revealed no significant relationship with age. This finding initially appears to support studies showing that sleep quality in older adults is not significantly different from middle-aged adults (e.g., Middelkoop et al., 1996). However, after controlling for gender, depression and SDB-risk, a significant relationship between age and global sleep quality emerged, such that increased age was associated with higher global scores on the PSQI (indicating poorer sleep quality). This would appear to suggest that these variables actually concealed age-related differences in global sleep scores, rather than contributed to them. In other words, age-related differences in global sleep quality were only apparent after depression, gender and SDB risk were controlled for. The size of the effect was small (Cohen, 1988), although significant. This finding is in direct contrast to previous research indicating that age differences in sleep quality disappear when controlling for variables such as depression (Roberts et al., 1999). However, Robert et al.'s sample comprised an older cohort only and not individuals 
across the lifespan. Therefore, the relationship between sleep and depression may change further as people reach older age. An examination of the relationship between age and PSQI factor scores (Cole et al., 2006), after controlling for gender, psychological factors and risk of SDB, also revealed interesting findings:

Firstly, Sleep Efficiency scores were positively associated with age, indicating increased time spent awake during the night in older participants. This is consistent with studies reporting reduced sleep efficiency and sleep duration as common sleep problems in older adults (Reid et al., 2006). However, again, this was not a robust finding given the small effect size. Secondly, Perceived Sleep Quality was not significantly associated with age, suggesting that participants’ ratings of their sleep quality did not differ much across the lifespan. Buysse et al. (1991) provided an explanation for this finding, suggesting that it could be because older adults adjust their expectations about their sleep quality, and are largely non-complaining about sleep changes. Thirdly, there was no relationship between age and Daily Disturbances, indicating that older adults did not report increased disruption to their daily lives as a result of poor sleep. This could be because older adults re-evaluate their expectations about how sleep impacts on their daily lives (Zilli et al., 2009), or they have reduced stressors and responsibilities, such as full-time work, study and childrearing, compared to younger adults, for whom the effects of poor sleep would, perhaps, be more pronounced.

In summary, current results revealed that sleep problems were common across the lifespan, and there was relatively little age-related difference in self-reported sleep quality across all indices of the PSQI. We also found that sleep-related risk factors such as gender, psychological symptoms and risk of SDB, although related to sleep quality, largely did not impact on the relationship 
between age and sleep quality. In other words, the modest age-related differences in self-reported sleep quality were not due to depressed mood, gender, or risk of SDB.

Altogether, these findings stand in contrast to studies of objective sleep that consistently report poorer sleep quality with advancing age (e.g., Espiritu, 2008; Unruh et al., 2008). Self-reports, however, are an important dimension of sleep because, firstly, clinicians use subjective complaints to determine the need and type of treatment used, and secondly, there is often a discrepancy between polysomnography and subjective sleep reports (Unruh et al., 2008). Given that there is a pervasive view that older adults typically experience more sleep difficulties than younger adults (Vitiello, 2006), our finding that poor self-reported sleep quality is not a prominent feature of older age suggests that reports of new sleep problems from older individuals merit further clinical investigation. This highlights the evaluation of self-reported sleep as a valuable and independent dimension of sleep assessment. 


\section{References}

Aloba, O. O., Adewuya, A. O., Ola, B. A., \& Mapayi, B. M. (2007). Validity of the Pittsburgh Sleep Quality Index (PSQI) among Nigerian university students. Sleep Med, 8, 266-270.

Amra, B., Farajzadegan, Z., Golshan, M., Fietze, I., \& Penzel, T. (2011). Prevalence of sleep apnea-related symptoms in a Persian population. Sleep and Breathing, 15(3), 425-429.

Beck, S. L., Schwartz, A. L., Towsley, G., Dudley, W., \& Barsevick, A. (2004). Psychometric evaluation of the Pittsburgh Sleep Quality Index in cancer patients. Journal of Pain and Symptom Management, 27, 140-148.

Buysse, D. J., Hall, M. L., Strollo, P. J., Kamarck, T. W., Owens, J., Lee, L., Reis, S. E., \& Matthews, K. A. (2008). Relationships between the Pittsburgh Sleep Quality Index (PSQI), Epworth Sleepiness Scale (ESS), and Clinical/Polysomnographic Measures in a Community Sample. Journal of Clinical Sleep Medicine, 4(6), 563-571. Correction in: Journal of Clinical Sleep Medicine (2010), February 15; 6(1), np.

Buysse, D. J., Reynolds, C. F., Monk, T. H., Hoch, C. C. et al. (1991). Quantification of subjective sleep quality in healthy elderly men and women using the Pittsburgh Sleep Quality Index (PSQI). Sleep: Journal of Sleep Research \& Sleep Medicine, 14(4), 331338.

Buysse, D. J., Reynolds iii, C. F., Monk, T. H., Berman, S. R., \& Kupfer, D. J. (1989). The Pittsburgh sleep quality index: A new instrument for psychiatric practice and research. Psychiatry Research, 28(2), 193-213.

Carpenter, J. S., \& Andrykowaski, M. A. (1998). Psychometric evaluation of the Pittsburgh 
Sleep Quality index. Journal of Psychosomatic Research, 45(1), 5-13.

Cohen, J. (1988). Statistical power analysis for the behavioral sciences (2nd ed.). Hillsdale, NJ: Erlbaum.

Cole, J. C., Motivala, S. J., Buysse, D. J. et al. (2006). Validation of a 3-factor scoring model for the Pittsburgh Sleep Quality Index in older adults. SLEEP, 29(1), 112-116.

Crawford, J. R., Garthwaite, P. H., Lawrie, C. J., Henry, J. D., MacDonald, M. A., Sutherland, J., \& Sinha, P. (2009). A convenient method of obtaining percentile norms and accompanying interval estimates for self-report mood scales (DASS, DASS-21, HADS, PANAS, and sAD. British Journal of Clinical Psychology, 48, 163-180.

Crawford, J. R., \& Henry, J. D. (2005). The short-form version of the Depression Anxiety Stress Scales (DASS-21): Construct validity and normative data in a large non-clinical sample. The British Journal of Clinical Psychology, 44(2), 227-239.

Doi, Y., Minowa, M., \& Tango, T. (2003). Impact and correlates of poor Sleep Quality in Japanese white-collar employees. American Academy of Sleep Medicine, 26(4), 467-471.

Espiritu, J. R. D. (2008). Aging-related sleep changes. Clinics in Geriatric Medicine, 24(1), 1-14.

Field, A. (2009). Discovering Statistics Using SPSS. (3rd ed.). London, UK: Sage Publications Ltd.

Fiske, A., Loebach Wetherell, J., \& Gatz, M. (2009). Depression in older adults. Ann Rev Clin Psychol, 5, 363-389.

Flemons, W. W., \& Tsai, W. (1997). Quality of life consequences of sleep-disordered breathing. Journal of Allergy and Clinical Immunology, 99(2), S750-S756. 
Foley, D. J., Monjan, A. A., Brown, S. L., Simonsick, E. M., Wallace, R. B., \& Blazer, D. G. (1995). Sleep complaints among elderly persons: An epidemiologic study of three communities. Sleep: Journal of Sleep Research \& Sleep Medicine, 18(6), 425-432.

Gentili, A., Weiner, D. K., Kuchibhatla, M., \& Edinger, J. D. (1995). Test-retest reliability of the Pittsburgh sleep quality index in nursing home residents. Journal of the American Geriatrics Society, 43, 1317-1318.

Grandner, M. A., Martin, J. L., Patel, N. P., Jackson, N. J., Gehrman, P. R., Pien, G., Perlis, M. L., Xie, D., Sha, D., Weaver, T., \& Gooneratne, N. S. (2012). Age and sleep disturbances among American men and women: data from the U.S. Behavioral Risk Factor Surveillance System. Sleep, 35(3), 395-406.

Hall, M., Buysse, D. J., Nowell, P. D., Nofzinger, E. A., Houck, P., Reynolds, C. F., \& Kupfer, D. J. (2000). Symptoms of stress and depression as correlates of sleep in primary insomnia. Psychosom Med,62, 227-230.

Harris, M., Glozier, N., Ratnavadivel, R., \& Grunstein, R. R. (2009). Obstructive sleep apnea and depression. Sleep Medicine Reviews, 13, 437-444.

Henderson, A. S., Jorm, A. F., Korten, A. E., Jacomb, P., Christensen, H., \& Rodgers, B. (1998). Symptoms of depression and anxiety during adult life: evidence for a decline in prevalence with age. Psychological Medicine, 28(6), 1321-1328.

Jorm, A. F. (2000). Does old age reduce the risk of anxiety and depression? A review of epidemiological studies across the adult lifespan. Psychological Medicine, 30(1), 11-22.

Knowles, J. B., \& MacLean, A. W. (1990). Age-related changes in sleep in depressed and 
healthy subjects. A meta-analysis. Neuropsychopharmacology: official publication of the American College of Neuropsychopharmacology, 3(4), 251-259.

Krishnan, P., \& Hawranik, P. (2008). Diagnosis and management of geriatric insomnia: A guide for nurse practitioners. Journal of the American Academy of Nurse Practitioners, 20(12), 590-599.

Lovibond, P. F., \& Lovibond, S. H. (1995). Manual for the Depression Anxiety Stress Scales. Sydney: Psychology Foundation.

Magee, C. A., Caputi, P., Iverson, D. C., \& Huang, X. F. (2008). An investigation of the dimensionality of the Pittsburgh Sleep Quality Index in Australian adults. Sleep and Biological Rhythms, 6(4), 222-227.

Mariman, A., Vogelaers, D., Hanoulle, I., Delesie, L., Tobback, E., \& Pevernagie, D. (2012). Validation of the three-factor model of the PSQI in a large sample of chronic fatigue syndrome (CFS) patients. J Psychosom Res, 72,111-113.

McCrae, C. S. (2009). Late-life comorbid insomnia: Diagnosis and treatment. The American Journal of Managed Care, 15(1), S14-S23.

Middelkoop, H. A. M., Smilde-van den Doel, D. A., Neven, A. K., Kamphuisen, H. A. C., \& Springer, C. P. (1996). Subjective sleep characteristics of 1,485 males and females aged 50-93: Effects of sex and age, and factors related to self-evaluated quality of sleep. The Journals of Gerontology Series A: Biological Sciences and Medical Sciences, 51A(3), M108-M115.

Netzer, N. C., Stoohs, R. A., Netzer, C. M., Clark, K., \& Strohl, K. P. (1999). Using the Berlin 
Questionnaire to identify patients at risk for the Sleep Apnea Syndrome. Annals of Internal Medicine, 131(7), 485-491.

O’Connor (2006). Do older Australians truly have low rates of anxiety and depression? A critique of the 1997 National Survey of Mental Health and Wellbeing. Australian and New Zealand Journal of Psychiatry 40(8), 623-631.

Ohayon, M. M., Carskadon, M. A., Guilleminault, C., \& Vitiello, M. V. (2004). Meta-analysis of quantitative sleep parameters from childhood to old age in healthy individuals: Developing normative sleep values across the human lifespan. Sleep, 27(7), 1255-1273.

Peppard, P. E., Szklo-Coxe, M., Hla, K., \& Young, T. (2006). Longitudinal Association of sleeprelated breathing disorder and depression. JAMA Internal Medicine, 166(6), 1709-1715.

Reid, K. J., Martinovich, Z., Finkel, S., Statsinger, J., Golden, R., Harter, K., \& Zee, P. C. (2006). Sleep: A Marker of Physical and Mental Health in the Elderly. American Journal of Geriatric Psychiatry 14(10), 860-866.

Roberts, R. E., Shema, S. J., \& Kaplan, G. A. (1999). Prospective data on sleep complaints and associated risk factors in an older cohort. Psychosomatic Medicine, 61(2), 188-196.

Sbarra, D. A., \& Allen, J. J. B. (2009). Decomposing depression: On the prospective and reciprocal dynamics of mood and sleep disturbances. Journal of Abnormal Psychology, 118(1), 171-182.

Spira, A. P., Beaudreau, S. A., Stone, K. L., Kezirian, E. J., Lui, L.Y., Redline, S., Ancoli-Israel, S., Ensrud, K., \& Stewart, A. (2012). Reliability and validity of the Pittsburgh Sleep Quality Index and the Epworth Sleepiness Scale in older men. Journals of Gerontology 
Series A: Biological Sciences and Medical Sciences, 67(4), 433-439.

Stein, M. B., Belik, S.L., Jacobi, F., \& Sareen, J. (2008). Impairment Associated With Sleep Problems in the Community: Relationship to Physical and Mental Health Comorbidity. Psychosomatic Medicine, 70(8), 913-919.

Taylor, D. J., Lichstein, K. L., Durrence, H., Reidel, B. W., \& Bush, A. J. (2005). Epidemiology of insomnia, depression, and anxiety. Sleep, 28(11), 1457-1464.

Unruh, M. L., Redline, S., An, M. W., Buysse, D. J., Nieto, F. J., Yeh, J. L., \& Newman, A. B. (2008). Subjective and objective sleep quality and aging in the Sleep Heart Health Study. J Am Geriatr Soc, 56, 1218-1227.

Vitiello, M. V. (2006). Sleep in normal aging. Sleep Medicine Clinics, 1, 171-176.

Vitiello, M. V., Moe, K. E., \& Prinz, P. N. (2002). Sleep complaints co segregate with illness in older adults: Clinical research informed by and informing epidemiological studies of sleep. Journal of Psychosomatic Research, 53(1), 555-559.

Voderholzer, U., Al-Shajlawi, A., Weske, G., Feige, B., \& Riemann, D. (2003). Are there gender differences in objective and subjective sleep measures? A study of insomniacs and healthy controls. Depression and Anxiety, 17(3), 162-172.

Walker, M. P., \& van der Helm, E. (2009). Overnight therapy? The role of sleep in emotional brain processing. Psychological Bulletin, 135(5), 731-748.

Wong, W. S., \& Fielding, R. (2011). Prevalence of insomnia among Chinese adults in Hong Kong: a population-based study. Journal of Sleep Research, 20, 117-126.

Zeitlhofer, J., Schmeiser-Rieder, A., Tribl, G., Rosenberger, A., Bolitschek, J., Kapfhammer, G., 
Saletu, B., Katschnig, H., Holzinger, B., Popovic, R., \& Kunze, M. (2000). Sleep and quality of life in the Austrian population. Acta Neurologica Scandinavica, 102(4), 249257.

Zilli, I., Ficca, G. \& Salzarulo, P. (2009). Factors involved in sleep satisfaction in the elderly. Sleep Medicine, 10(2), 233-239. 
Table 1. Descriptive statistics for the demographics questionnaire, including Berlin Questionnaire and Depression Anxiety and Stress Scale 21 (DASS-21; $N=582$ )

\begin{tabular}{lc}
\hline \multicolumn{1}{c}{ Variable } & N: \%, Median [IQR], range or \% \\
\hline Age in years & 33.30 [30], 18-89 \\
& $257: 43.6 \%$ \\
$18-29$ & $78: 13.4 \%$ \\
$40-39$ & $71: 12.2 \%$ \\
$50-59$ & $69: 11.9 \%$ \\
$60-69$ & $63: 10.8 \%$ \\
$70+$ & $47: 8.1 \%$ \\
Gender: Males & $154: 26.5 \%$ \\
Body Mass Index ${ }^{1}$ & 23.66 [6.02], $15.62-44.41$ \\
Risk of Sleep Disordered Breathing & $125: 21.1 \%$ \\
(Berlin Questionnaire) & $391: 67.2 \%$ \\
Completed tertiary education & $316: 54.3 \%$ \\
Currently employed & $164: 28.2 \%$ \\
Student & $127: 21.8 \%$ \\
Seen a Doctor for mental health issues & 4 [8.5], 0-42 \\
DASS-21 - Depression & $2[6], 0-42$ \\
DASS-21 - Anxiety & 8 [10], 0-36 \\
DASS-21 - Stress & \\
\hline
\end{tabular}

Note: ${ }^{1} N=532$ due to missing data; DASS-21 = Depression Anxiety Stress Scale- 21 item version (Lovibond \& Lovibond, 1995). 
Table 2. Descriptive statistics for the Pittsburgh Sleep Quality Index (PSQI; N=582)

\begin{tabular}{llccc}
\hline & Global PSQI & $\begin{array}{c}\text { Cole et al.'s factor 1: } \\
\text { Sleep Efficiency }\end{array}$ & $\begin{array}{c}\text { Cole et al.'s factor 2: } \\
\text { Perceived Sleep } \\
\text { Quality }\end{array}$ & $\begin{array}{c}\text { Cole et al.'s factor 3: } \\
\text { Daily Disturbances }\end{array}$ \\
\hline All ages & $6.4(3.3), 0-17$ & $1.2(1.4), 0-5$ & $2.0(1.2), 0-6$ & $1.4(0.6), 0-3.6$ \\
$18-29$ & $6.1(2.9), 0-14$ & $0.9(1.3), 0-5$ & $2.0(1.1), 0-5.1$ & $1.3(0.5), 0-3.6$ \\
$30-39$ & $6.6(3.5), 1-15$ & $1(1.3), 0-5$ & $2.2(1.2), 0-5.5$ & $1.4(0.6), 0-2.9$ \\
$40-49$ & $6.4(3.7), 1-16$ & $1.3(1.6), 0-5$ & $1.9(1.2), 0-4.4$ & $1.4(0.6), 0-3.1$ \\
$50-59$ & $6.8(3.4), 2-17$ & $1.3(1.4), 0-5$ & $2.1(1.2), 0-6$ & $1.4(0.5), 0-2.4$ \\
$60-69$ & $6.4(3.7), 2-16$ & $1.4(1.4), 0-5$ & $1.8(1.2), 0-5.1$ & $1.3(0.5), 0-3$ \\
$70+$ & $6.9(3.8), 2-15$ & $1.5(1.5), 0-4.3$ & $1.9(1.3), 0-5.1$ & $1.4(0.5), 0.7-2.9$ \\
\hline
\end{tabular}

Note: ${ }^{1}$ Cole et al. (2006). 
Table 3. Correlation matrix (Spearman correlation coefficients) for age, sleep quality (PSQI), and psychological symptoms (DASS-21)

\begin{tabular}{lcccc}
\hline & Age & $\begin{array}{c}\text { DASS- } \\
\text { Depression }\end{array}$ & $\begin{array}{c}\text { DASS- } \\
\text { Anxiety }\end{array}$ & $\begin{array}{c}\text { DASS- } \\
\text { Stress }\end{array}$ \\
\hline Age & - & $-.17 \ddagger$ & $-.09 \dagger$ & $-.12 \ddagger$ \\
PSQI Global & .03 & $.33 \ddagger$ & $.12 \ddagger$ & $.11 \ddagger$ \\
Cole's Factor 1: Sleep efficiency $^{1}$ & $.16 \ddagger$ & $.15 \ddagger$ & .04 & .02 \\
Cole’s Factor 2: Perceived sleep quality $^{1}$ & -.05 & $.23 \ddagger$ & .07 & .07 \\
Cole's Factor 3: Daily disturbances $^{1}$ & -.02 & $.40 \ddagger$ & $.18 \ddagger$ & $.15 \ddagger$ \\
\hline
\end{tabular}

Note: $\dagger p<.05$, $p p<.01$; Higher scores on all measures indicate greater disturbance; ${ }^{1}$ Cole et al. (2006). 
Table 4. Regression analyses for all indices of sleep quality (PSQI)

\begin{tabular}{|c|c|c|c|c|c|}
\hline Dependent variable & Covariate(s) & Predictor & $\begin{array}{l}\text { Beta }(95 \% \mathrm{CI}) \text { for } \\
\text { predictor }\end{array}$ & $\begin{array}{c}\text { R square } \\
\text { covariates only } \\
\text { (Step 1) }\end{array}$ & $\begin{array}{l}\text { R square change } \\
\text { after predictor } \\
\text { added (Step 2) }\end{array}$ \\
\hline PSQI Global $^{1}$ & $\begin{array}{c}\text { Gender } \dagger \text {, Risk of SDB } \dagger, \text { DASS- } \\
\text { Depression } \ddagger\end{array}$ & Age & $1.00(0-.03) \dagger$ & $.15 \ddagger$ & $.01 \dagger$ \\
\hline Factor 1: Sleep Efficiency ${ }^{1,2}$ & $\begin{array}{c}\text { Risk of SDB } \uparrow, \text { DASS- } \\
\text { Depression } \neq\end{array}$ & Age & $.17(.01-.02) \ddagger$ & $.06 \ddagger$ & $.03 \ddagger$ \\
\hline Factor 2: Perceived Sleep Quality ${ }^{1,2}$ & Gender $†$, DASS-Depression $\ddagger$ & Age & $-.02(-.01-.01)$ & $.06 \ddagger$ & $<.01$ \\
\hline Factor 3: Daily Disturbances ${ }^{1,2}$ & $\begin{array}{c}\text { Gender } \dagger \text {, Risk of SDB } \ddagger \text {, DASS- } \\
\text { Depression } \ddagger\end{array}$ & Age & $.01(0)$ & $.19 \ddagger$ & $<.01$ \\
\hline
\end{tabular}

Note. $\dagger=p<.05$, $\neq=p<.01$; Significance values for the covariates are reported from the final model (Step 2); ${ }^{1}$ Higher scores indicate greater disturbance; ${ }^{2}$ Cole et al. (2006). 